\title{
Les crues historiques de la Seine à Paris
}

\author{
par A. Goubet \\ Ingénieur général des Ponts \& Chaussées
}

\section{I a POURQUOI LES “CRUES HISTORIQUES” ET POURQUOI "À PARIS”}

Entre 1808 et 1909, Paris n'a connu qu'une crue importante, celle de 1876 dont la durée de retour est à peu près vingtennale. Pendant la même période, la Loire voisine a connu trois crues "séculaires". Dans un cas comme dans l'autre, seul l'examen des événements observés pendant la plus longue période historique possible permet de définir la durée de retour des grandes crues.

Par ailleurs, la chronique des crues à Paris est très différente de celle de ses affluents : la crue de février/mars 1784 constitue le record toujours inégalé pour l'Oise et la Marne ; elle a été à peu près vingtennale à Paris. De même les crues de 1836 et 1856 n'ont jamais été dépassées en certains points du bassin de l'Yonne ; elles ont été insignifiantes à Paris. Et une étude limitée à Paris constitue déjà une tâche de grande ampleur.

\section{LE CADRE PHYSIQUE : LE LIT DE LA SEINE À PARIS}

\subsection{Tracé en plan}

Il convient d'oublier les affirmations souvent répétées sur la Seine, fleuve large, baignant des rives marécageuses, et étranglée par les Hommes entre des quais rapprochés bordant un lit dont le fond se surélevait chaque fois qu'un pont s'écroulait. Cette vision est très largement erronée.

Jadis, la Seine faisant un grand demi-cercle dans Paris, baignait le pied de toutes les collines de Belleville à Chaillot. A la suite d'un phénomène non précisé, mais qui pourrait être un captage dans lequel la Bièvre aurait joué un rôle, le fleuve a adopté son lit actuel. Sa longueur s'est raccourcie d'un tiers et sa pente a augmenté de moitié entre le bassin de l'Arsenal et Chaillot. Le cœur de Paris s'est donc installé, puis développé à l'emplacement d'une anomalie géographique.

Ce déplacement était déjà cité en 1651 comme un fait bien connu (et il semblerait qu'il ait également été connu dès 1512).

Dans le centre de Paris, la Seine a de ce fait un lit naturellement étroit : entre le pied de la Montagne Ste Geneviève et les buttes du bord de la rive droite (1) (Monceau St Gervais et butte, supprimée par Haussmann, sur laquelle se trouvait la tour St Jacques) il n'y a que 7 à $800 \mathrm{~m}$ qui étaient la largeur maximum que pouvait occuper la rivière lors de ses plus grandes crues.

Entre ces deux berges se trouvait l'Ile de la Cité. Quelle était sa hauteur? On ne peut que se référer à des îles voisines qui n'ont pas été occupées à l'origine par l'homme et qui étaient vraisemblablement plus basses.

L'île St Louis, dont certains affirment qu'elle était recouverte par les eaux chaque hiver, n'était en fait recouverte qu'exceptionnellement. A propos de la crue de juin 1428 , on peut lire "[l'eau] allait jusqu'à St Esprit... L'lle de St Louis fut toute couverte". Cette phrase montre que la submersion de l'île correspond à une crue particulièrement forte (plus que décennale). D'ailleurs les premières habitations de l'île furent construites dès le début du XVIIème siècle en son centre mais avant le début des travaux d'endiguement.

(1) Habitées dès l'époque romaine.

Until now, it has just been tried to reconstitute the maximum water gauge at the time of the Seine floods since 1732, 1649 or 1600 . But the flow rates were not associated with these water gauges. In reality, a careful examination of all the past look-outs permitted to collect one or two centuries older data and to try to model the flow variations and estimate the flow rates from 1600 . 
L'île Louviers n'a pas été totalement couverte en $1740^{(2)}$ et avait son sommet à plus de $8 \mathrm{~m}$ au-dessus de l'étiage avant son rattachement au rivage (mais je pense qu'elle avait été surélevée artificiellement).

L'île de Neuilly, avant que Peyronnet ne remodelle la Seine, s'élevait à environ $5 \mathrm{~m}$ sur l'étiage (pour $200 \mathrm{~m}$ de large).

On peut donc penser que l'île de la Cité, la seule à avoir été habitée dans l'antiquité, était plus haute (et plus large) et pouvait avoir eu son sommet à 6,5 ou $7 \mathrm{~m}$ environ au-dessus de l'étiage. Cela la mettait à l'abri des crues vingtennales. De modestes travaux de surélévation localisés (ou de construction sur pilotis) pouvaient en faire un endroit très habitable.

\section{- 2.2. Profil en long}

Le lit de la Seine avait en son état naturel un profil assez complexe à la traversée du Paris du XVIIIème siècle.

A son extrémité aval se trouvait le banc de l'Aiguillette, le (ou un des) principal haut fonds entre Paris et Rouen. La profondeur du lit à l'étiage était inférieure à $1 \mathrm{~m}$. Mais un peu à l'amont le fond de la Seine s'approfondissait à $5 \mathrm{~m}$ entre le Pont Royal et le Pont de la Concorde, ainsi que sous le pont Royal, à 3,5 au pont de Carrousel puis il se relevait progressivement avant le Pont Neuf ; sa profondeur à l'étiage était de $1 \mathrm{~m}$ environ dans le bras sud de l'île de la Cité, sous le pont au Change, sous le pont de la Tournelle et vers le pont d'Austerlitz.

En revanche, il y avait un approfondissement important et localisé en face de la Grève (la place de l'Hôtel de Ville), peut-être dû au remous qui se formait à la jonction du bras nord de l'Ile St Louis et du bras situé entre celle-ci et l'île de la Cité.

Dans ces conditions, à l'étiage le niveau de la Seine était quasi horizontal entre le banc de l'Aiguillette et le Pont du Carrousel puis présentait une forte pente plus à l'amont, jusqu'au pont d'Austerlitz.

\subsection{Rives de la Seine}

On a dit que la Seine avait des rives basses et marécageuses : dans la traversée de Paris, comme à l'amont ou à l'aval (et ainsi qu'on pouvait le vérifier encore du début du XIXème siècle) les terres qui bordaient la Seine l'élevaient, comme les îles, à 5 - 6 ou $7 \mathrm{~m}$, exceptionnellement davantage, au-dessus de l'étiage et étaient submergées par les crues à peine décennales aux points bas, vingtennales aux points hauts. Le reste du temps le sol était émergé ; sa composition géologique est extrêmement variable mais comporte beaucoup de sable qui assurait un bon drainage vers le fleuve et rend peu vraisemblable la présence de grandes zones marécageuses.

On peut remarquer que le resserrement constaté au droit de l'lle de la Cité est une particularité locale. En amont et en aval on peut encore trouver des buttes en bordure de Seine sur une rive (Chaillot, Butte aux Cailles), mais sur la rive opposée existe une plaine basse et aisément inondable (Champs de Mars, Bercy) qui autorisait un large débordement lors des très grandes crues. C'est cette singularité, peut-être ajoutée aux dimensions de l'Ile de la Cité, qui explique vraisemblablement le choix du site et son importance stratégique (commande de l'emplacement où il était le plus aisé de construire un pont).

\section{- 2.4. Cas particulier de l'ancien lit de la Seine}

L'ancien lit a vraisemblablement été comblé par les matières en suspension apportées par la Seine et qui se sont déposées dans les endroits où la vitesse du courant était le plus faible ; elles contenaient aussi certainement des matières organiques. Le niveau du sol y était resté bas (inférieur à celui des berges de l'ancienne Seine) et proche de la nappe phréatique (dont le niveau était commandé par celui de la Seine dans son nouveau lit). Toutes les conditions étaient réunies pour donner naissance à une vaste zone marécageuse qui a gêné l'établissement des routes en direction du nord, de l'est ou de l'ouest, lesquelles ont été établies sur remblai et comportaient éventuellement des ponts.

(2) La troisième crue par son importance depuis 4 siècles.

\section{III $\square$ LES COTES ATTEINTES PAR LES CRUES}

\subsection{Les échelles}

La première échelle fut installée avant 1719 (peut-être en 1714) au Pont Royal sur la première pile au nord. Elle avait une utilité essentiellement pratique : informer les mariniers qui effectuaient le trajet Rouen-Paris de la profondeur d'eau sur le banc de l'Aiguillette. La plus ancienne cote connue sans aucune ambiguïté à Paris est celle de l'étiage de septembre 1719 à cet emplacement. Très tôt ensuite cette échelle fut doublée par une seconde installée sur la première pile au sud.

Un peu après 1719 une autre échelle fut installée dans l'angle du quai et de la culée rive droite, à l'amont du Pont de la Tournelle. La cote y a été relevée pratiquement sans interruption depuis le ler janvier 1732 jusqu'en 1852. Pendant plus d'un siècle cette échelle fut la référence non seulement pour les études hydrologiques mais aussi pour tout ce qui avait un rapport avec la Seine (quai, voirie, égout...).

Une troisième échelle fut mise en place au Pont Notre Dame en 1769 mais je n'ai jamais trouvé de référence à une cote qui y aurait été relevée.

Une quatrième échelle suivit en 1787 en rive droite à l'aval du pont de la Concorde alors en construction mais il semble qu'elle n'ait eu qu'une durée de vie brève. En effet, pour éviter l'influence des ponts sur les cotes lues aux échelles fixées sur ces ouvrages, on avait, semble-t-il, installé l'échelle à quelque distance en aval, dans une zone qui n'était pas encore dotée de quai. La construction du "sequanomètre", envisagée et commencée en 1787, semble toutefois ne jamais avoir été terminée et la réalisation du quai une vingtaine d'années plus tard a conduit à reporter l'échelle sur le pont. Même si les zéros étaient strictement à la même cote, les deux séries de valeurs relatives à l'échelle de la Concorde ne sont donc pas tout à fait homogènes. De toute façon les hydrologues du XIXème siècle n'ont que très rarement fait référence aux cotes du pont de la Concorde.

Enfin, on cite l'existence en 1789 d'une autre échelle sur la seconde pile (en partant du nord) et en aval du pont de la Tournelle. ainsi qu'une dernière à l'estacade de l'île Louviers. Mais je ne connais non plus aucun relevé effectué à ces deux emplacements.

\subsection{Les marques et repères}

Si depuis 1732 on connaît les cotes atteintes par toutes les crues ${ }^{(3)}$, les renseignements sont moins complets pour les crues antérieures. Les analyses faites au XVIIIème siècle sur les marques gravées en différents endroits lors des crues les plus importantes ont permis de répertorier les crues vingtennales (environ) jusque vers 1600 .

Mais l'expérience montre que, au moins à Paris, les marques de crue disparaissent rapidement. Pour la période antérieure à 1600 on dispose d'autres types d'information essentiellement liés à l'existence de la Grève.

La Grève (ou la place de Grève) était, à l'intérieur de la ville ancienne, le point le plus bas ; elle se situait devant la façade de l'Hôtel de Ville mais était beaucoup plus petite que la place actuelle (elle occupait la partie Est la plus proche de la Seine). Cet emplacement n'a jamais été bâti (trop facilement inondable) et servait de place de marché ; le débordement de la Seine sur la partie la plus proche de la rivière commençait avant même la crue décennale.

L'Hôtel de Ville reconstruit sous François ler comportait vers le sud un porche, l'arcade St Jean, qui surmontait un passage public : il comportait au nord un second porche qui donnait accès à la Chapelle de l'Hospice de St Esprit. L'eau commençait à atteindre le premier (pour une cote qui est à peu près connue) puis le second et les chroniqueurs citaient toujours cette référence ${ }^{(4)}$. Avant 1540 ces repères précis n'existaient pas et l'on se contentait d'écrire "l'eau atteint l'hôtel de Ville" ou pour les crues plus importantes l'eau dépasse la Grève et atteint telle ou telle rue située plus au nord.

(3) Avec un certain flou en ce qui concerne la crue de 1740 qui a certainement dépassé le sommet de l'échelle du Pont Royal et vraisemblablement celle du pont de la Tournelle.

(4) "l'eau atteignit le Passage St Jean" ou plus rarement "l'eau atteignit le Saint Esprit". 
L'on a aussi beaucoup parlé de la Croix dressée en place de Grève. Cette croix a existé pendant plusieurs siècles ; elle était placée au sommet d'une pyramide en marches d'escalier au bord sud de la place de Grève et l'on pouvait se référer au nombre de marches noyées. Mais j'ai beaucoup moins confiance dans cette indication depuis que j'ai constaté que, selon les historiens, la pyramide avait tantôt huit marches et tantôt 13 marches ! (Elle a dû être supprimée en 1673, son pied avait pu être antérieurement remblayé).

Enfin, s'il s'agit de repérer les crues de l'ordre de celles de 1658 ou 1910 on peut se référer à une dernière particularité. Les débordements de 1658 ont complètement entouré Paris par le nord le long de l'ancien bras de Seine (à l'exclusion du sol des grandes routes qui rayonnaient depuis Paris) ; ceux de 1910 l'auraient peut-être fait si le sol n'avait été relevé entre temps ; on peut rechercher dans les chroniques celles où se trouve mentionné un tel phénomène ; elles sont très rares et peuvent correspondre à des débits et des cotes inférieurs puisque les sols étaient vraisemblablement plus bas ; mais aussi longtemps qu'on n'en trouve pas mention, on peut être sûr que la crue était inférieure aux deux citées ci-dessus. J'ai dû remonter six siècles en arrière pour trouver une mention qui permet de supputer un tel contournement. Les crues de 1658 et 1910 sont donc les deux plus importantes depuis 6 siècles.

\section{- 3.3. Les résultats relatifs aux cotes}

Une analyse statistique portant sur 49 ans (1777-1825) donne les résultats suivants (cotes à l'échelle de la Tournelle rapportées à l'étiage de référence observé en 1719 et 1840 - il ne s'agit pas de la plus basse cote, qui a été relevée en 1767) :

médiane $0,94 \mathrm{~m}$

moyenne $1,25 \mathrm{~m}$ (peut varier selon les années de 0,66 à 2,34 m)

Une analyse portant sur la période 1732-1854 (à l'exception d'une année) montre que le maximum annuel est à la même échelle (5): une année sur deux $<4,30 \mathrm{~m}$ une année sur dix $\quad<5,85 \mathrm{~m}$

Les crues supérieures à $7,00 \mathrm{~m}$ et postérieures à 1600 sont les suivantes (sachant que les crues antérieures à 1740 ne sont connues que de façon approximative) :

$\begin{array}{llll}01.03 .1658 & 8,80 & 12.03 .1711 & 7,60 \\ 26.01 .1910 & 8,42 & \text { mars } 1690 & 7,60 \\ 25.12 .1740 & 8,10 & 03.01 .1802 & 7,45 \\ 25.01 .1651 & 7,80 & 06.01 .1924 & 7,18 \\ 01.02 .1649 & 7,70 & 09.02 .1764 & 7,00\end{array}$

soit 10 crues en 400 ans.

Il n'est pas totalement impossible qu'une crue comprise entre 7,00 et 7,50 ait été omise entre 1600 et $1700^{(6)}$.

\subsection{Informations relatives aux débits}

\section{Influence des "ponts" parisiens vers 1750}

Lors de la crue exceptionnelle de 1740 (la troisième en 4 siècles), le pont Royal était le plus à l'aval de la capitale. L'urbanisation proche des berges s'arrêtait à une faible distance à l'aval (Palais Bourbon en rive gauche, jardin des Tuileries en rive droite). La cote atteinte à l'échelle du pont Royal correspondait donc, à 1 ou 2 centimètres près, à l'état naturel de la Seine. A l'amont se développait sur quelques kilomètres la partie "étranglée" du lit de la Seine jusqu'au bassin de l'Arsenal (mais les quais s'arrêtaient, vers l'amont, dès le milieu de l'île de la Cité, aussi bien dans l'Ile que sur les berges).

La comparaison des cotes atteintes en 1740 et lors des crues suivantes de 1751 et 1764 , sensiblement plus faibles (de 78 et $57 \mathrm{~cm}$ à

(5) Sur 120 maxima annuels, 18 sont observés en décembre, 34 en janvier, 30 en février et 26 en mars (surtout pendant la première quinzaine)

(6) On pourrait dresser un tableau des crues supérieures à $6,50 \mathrm{~m}$ depuis

1732 mais pas depuis 1600 , des crues anciennes de l'ordre de $6,5 \mathrm{~m}$ étant certainement tombées dans l'oubli le plus total. la sortie aval de Paris) montre que la dénivellation entre l'amont et l'aval était plus élevée respectivement de $5 \mathrm{~cm}$ et $45 \mathrm{~cm}$ en 1764 et 1740 qu'en 1751. Ces différences doivent être comparées à la dénivellation totale qui entre Chaillot et le bassin de l'Arsenal était de $1,45 \mathrm{~m}$ en 1764. Autrement dit, entre ces deux points, la dénivellation était de :

$140 \mathrm{~cm}$ en 1751 pour un débit de l'ordre de $\quad 1850 \mathrm{~m}^{3} / \mathrm{s}$ $145 \mathrm{~cm}$ en $1764 \quad$ * $1950 \mathrm{~m}^{3} / \mathrm{s}$

$185 \mathrm{~cm}$ en $1740 \quad$. $\quad 2150 \mathrm{~m}^{3} / \mathrm{s}$

Ces dénivellations peuvent paraître considérables pour une distance de l'ordre de $5500 \mathrm{~m}$, s'agissant d'un fleuve de plaine, et l'on peut penser que même pour une crue de $1800 \mathrm{~m}^{3} / \mathrm{s}$ l'étranglement de Paris provoque, en amont une surélévation considérable ${ }^{(7)}$. Il n'en est rien.

La différence d'altitude entre les zéros des deux échelles du pont Royal et du pont de la Tournelle est de $1,77 \mathrm{~m}$; comme les cotes lues au pont Royal sont toujours plus élevées que celles relevées à l'échelle du pont de la Tournelle, les dénivellations de la surface de l'eau sont plus faibles : à l'étiage $1,04 \mathrm{~m}$ au VIIIème siècle (de 1719 à 1800 environ) - 1,20 en 1840 .

Ces valeurs relatives à l'étiage sont peut-être surévaluées artificiellement car il est possible qu'à l'époque les ouvrages qui encombraient le pont Notre Dame ainsi que les fondations des piles du pont de la Tournelle aient provoqué une surélévation d'une dizaine de centimètres. Néanmoins une dénivellation à l'étiage de $1 \mathrm{~m}$ sur 2,5 km est quelque chose d'anormal pour la Seine et qui s'explique par deux motifs :

— nous avons déjà dit qu'à l'étiage le plan d'eau était quasi-horizontal entre le banc de l'Aiguillette et le Pont Royal ; la dénivellation entre les deux échelles pourrait donc être associée à une longueur de $4,5 \mathrm{~km}$ (distance entre le banc de l'Aiguillette et l'Arsenal),

- la pente "normale" de 15 à $20 \mathrm{~cm}$ serait à envisager le long de l'ancien bras de Seine qui avait atteint son profil d'équilibre mais qui avait $2 \mathrm{~km}$ environ de plus que le nouveau. La différence de cote de $1 \mathrm{~m}$ environ entre l'entrée et la sortie de l'ancien bras de Seine devient alors tout à fait normale.

Puisque la forte pente constatée à l'étiage entre les 2 échelles de référence résulte, pour une part, de la très faible pente à l'aval du pont Royal, lorsque le débit augmente la pente s'accroît sensiblement entre le banc de l'Aiguillette et le Pont Royal et, en contrepartie, diminue à l'amont. C'est bien ce qui est constaté dans le tableau 1 où figurent des cotes relevées en 1807 en 4 emplacements : les 2 échelles mentionnées ci-dessus, l'aval du pont d'Austerlitz (c'est-àdire un peu à l'amont de l'Arsenal) et à la pompe à feu de Chaillot (c'est-à-dire à peu près le banc de l'Aiguillette.

\begin{tabular}{|c|c|c|c|}
\hline $\begin{array}{c}\text { cote au } \\
\text { Pont Royal }\end{array}$ & $\begin{array}{c}\text { dénivellation } \\
\text { Austerlitz- } \\
\text { Chaillot }\end{array}$ & $\begin{array}{c}\text { dénivellation } \\
\text { Tournelle- } \\
\text { Royal }\end{array}$ & $\begin{array}{c}\text { débit } \\
\text { (environ) }\end{array}$ \\
\hline 0,73 & - & 1,04 & 50 (étiage) \\
\hline 2,24 & 1,46 & 0,99 & 300 \\
\hline 2,58 & 1,39 & 0,97 & 370 \\
\hline 2,69 & 1,39 & 0,97 & 390 \\
\hline 5,15 & 1,28 & 0,85 & 1000 \\
\hline 5,35 & 1,31 & 0,87 & 1050 \\
\hline 5,92 & 1,35 & 0,90 & 1200 \\
\hline 6,58 & 1,45 & 0,96 & 1400 \\
\hline 7,08 & 1,50 & 1,08 & 1650 \\
\hline 7,30 & 1,43 & 1,13 & 1850 \\
$(\max 1807)^{(8)}$ & & & \\
\hline
\end{tabular}

Tableau 1. - Cotes relevées en 1807 et débit.

(7) La pente de la Seine, variable selon les tronçons est généralement de 15 à $20 \mathrm{~cm}$. (8) Le maximum de la crue de 1807 est très voisin de celui de 1751 ; entre les 2 dates, deux nouveaux ponts avaient été réalisés à l'aval (passerelle des Arts et pont de la Concorde), dont le débouché était large. La place de la Concorde avait été aménagée. Je ne sais pas si la pompe de la Samaritaine avait déjà été supprimée en 1807. 
Comme l'échelle de la Tournelle est en amont du pont (dans l'angle du quai et de la culée) et celle du pont Royal à l'aval (en plus sur une pile) les différences d'altitude relevées aux échelles en crue sont sensiblement supérieures à celle de la surface de la rivière. On peut donc estimer que la surélévation à l'amont imputable à la présence de la ville était au milieu du XVIIIème siècle et au début du XIXème de $15 \mathrm{~cm}$ environ pour un débit de $1950 \mathrm{~m}^{3} / \mathrm{s}$ et atteignait $60 \mathrm{~cm}$ pour un débit de $2150 \mathrm{~m}^{3} / \mathrm{s}$ (le débit maximum pendant la période 1700-1900).

On ne peut pas évaluer de façon précise la situation correspondant à la crue de 1658 pour laquelle on ne possède aucune cote indiscutable dans Paris mais un rapport établi à l'époque permet d'estimer la surélévation à 2 pieds $(65 \mathrm{~cm})$... à un pied près !!

\subsection{Comment l'encombrement du lit de la Seine a-t-il évolué depuis 1600 ?}

Il a été maximal de 1670 (installation de la pompe élévatrice du pont Notre Dame) à 1800 . Il a connu une nette aggravation entre 1600 et 1670 , une petite amélioration entre 1800 et 1850 , puis une amélioration plus nette entre 1850 et 1900 .

\section{De 1600 à 1670}

En 1600, il y avait à l'aval le Pont Neuf (en voie d'achèvement) puis deux ponts sur chacun des deux bras de l'île de la Cité. Il y avait déjà des murs de quai sur les deux berges entre ces ponts et même à l'aval.

En 70 ans on a ajouté :

- la pompe de la Samaritaine qui a obstrué une des arches du Pont Neuf (qui avait été toutefois largement dimensionné),

- deux ponts sur le petit bras (le Pont au Double construit en 1632 , essentiellement pour supporter de nouveaux bâtiments de l'Hôtel Dieu, et le pont St Charles, pont privé, interne à l'Hôtel Dieu commencé en 1646).

On a également :

- permis à l'Hôtel Dieu de construire sur la rive gauche un nouveau bâtiment qui empiétait en fait d'une dizaine de mètres sur la Seine,

- autorisé sur la rive droite la construction d'une rangée de maisons le long du quai des Gesvres en surplomb sur la Seine entre les deux ponts préexistants (elle était supportée par une voûte sur piliers construite sur la Seine) vers 1640 ,

- installé une pompe élévatrice qui a pratiquement obstrué 2 passes du pont Notre Dame et encombré partiellement à l'étiage deux autres passes,

- loti l'Mle St Louis en la bordant de quais (ce qui ne présentait aucun inconvénient en ce qui concerne l'écoulement des crues) et en construisant 3 nouveaux ponts (Marie, de la Tournelle et passerelle en bois entre les 2 îles)

\section{De 1670 à 1800}

Voici comment la situation était décrite en 1760 en ce qui concerne l'île de la Cité.

Sur le bras nord, on a "laissé construire le quai de Gêvres", sur le lit même de la rivière, en avançant de "la valeur d'une arche et d'une pile des ponts au Change et de Notre Dame ;" car les piles de ce quai, "le long mur qui lui sert de culée, lequel bouche, du côté d'amont, un tiers de la première arche du pont" Notre Dame ; les reins de cette longue voûte, qui avec ses piles ne forment presque qu'un long tuyau "qui va en diminuant du côté du pont au Change", formant un obstacle considérable au passage de l'eau, "lequel joint à ceux que présente la digue que l'on a faite devant les deux premières arches du pont" "Notre Dame, pour envoyer l'eau aux roues des pompes quand la rivière est basse", les deux charpentes "de ces machines, les ailerons qui mènent l'eau dans les coursiers des roues", le puisard des pompes qui "est entre deux", les roues, leurs châssis et les pales ; tous ensemble forment certainement autant" d'obstacles dans tous les temps, que si on bouchait entièrement une arche et plus des six qu'à ce pont ; "joignant à cela l'obstacle indispensable des piles et des brèches", le tout fait au moins le tiers du "passage de ce bras de la rivière".
La situation n'était pas meilleure sur le bras sud où l'on a construit, en même temps que le pont au Double, "le bâtiment qui est le long de la rue de la Bucherie", embrassant une arche et une pile de ce "nouveau pont", de la même manière que le fait le quai de Gêvres pour le pont Notre Dame, si ce n'est "que le passage de l'eau était bien moins libre ici", étant obligée de sortir par deux petites ouvertures" qu'on pratiqua sur la face du bâtiment, n'ayant point d'issue au bout, où est le Petit-châtelet ; et pour "rendre cette arche tout à fait inutile au passage de l'eau", on l'a murée du côté d'amont, et fait un "batardeau par devant rempli de terre de 12 à 15 pieds de hauteur ou davantage".

La description, faite en 1864 , de la situation antérieure aux travaux du Second Empire n'est pas plus optimiste :

- le pont Notre Dame avait 6 arches dont une qui alimentait les cagnards (c'est-à-dire la voûte qui supportait les maisons du qua des Gesvres ; les maisons avaient été abattues mais la voûte subsistait) ; deux arches étaient obstruées par les roues de la pompe à eau, une autre par un déversoir (pour alimenter les roues lors de l'étiage) ; 2 passes seulement étaient libres et l'on constatait une chute de 0,3 à $0,4 \mathrm{~m}$ à l'étiage ${ }^{(9)}$

- dans l'autre bras existaient deux banquettes de halage et de contre-halage qui obstruaient la moitié de la section jusqu'à une hauteur de 4 mètres.

\section{De 1800 à 1850}

La situation évolue peu, mais plutôt favorablement : pour la première fois on envisage de mettre Paris à l'abri des crues au moyen de rangées continues de quais de hauteur homogène. Ces quais améliorent de quelques pour cent la vitesse moyenne du courant et peuvent provoquer à débit égal une baisse du plan d'eau d'un décimètre.

On supprime également la pompe de la Samaritaine. En revanche, on construit de nouveaux ponts mais avec des arches beaucoup plus larges qu'antérieurement. Il doit y avoir compensation.

\section{De 1850 à 1910}

Les responsables de l'époque semblent avoir perdu de vue le problème des crues (on avait même trouvé la cause de l'amélioration sensible du régime de la Seine : la déforestation (sic !!). Par contre on veut rendre la Seine navigable à la traversée de Paris tandis qu'Haussmann refait la voirie (et donc les ponts).

La totalité des ponts des 2 bras de l'île de la Cité sont remaniés : - sur le petit bras en rive gauche, le Pont St Charles disparaît ; le Petit Pont et le pont au Change sont remplacés par des ouvrages sans pile intermédiaire, le pont St Michel en perd une : on passe dans ce bras de 8 ou 9 larges piles à 2 piles plus minces ;

- sur le grand bras, en rive droite, les "cagnards" sont supprimés et le pont au Change passe de 7 arches à trois. En revanche, la transformation du Pont Notre Dame est moins heureuse : pour abaisser la chaussée de 2,70 m (Haussmann aimait les droites aussi bien en alignement qu'en profil en long) on se contente de reconstruire les voûtes en les abaissant d'autant ! Mais les pompes élévatrices disparaissent en 1858 ;

- et même si le pont St Louis a paru bien bas en 1910, il dégageait davantage le lit que les 6 piles du pont en bois du XVIIIème siècle.

Mais surtout on a très sensiblement approfondi le lit de la Seine. Le fond du lit ne s'était pas relevé depuis les Romains ; au contraire il s'était plutôt légèrement abaissé (ce qui est normal : le rétrécissement du lit comme la présence des piles ne pouvaient qu'accroître l'érosion). Mais à partir de 1850 les dragages interviennent de façon massive : - le niveau de l'étiage avait baissé de $60 \mathrm{~cm}$ entre 1840 et 1868 l'aval du Port à l'Anglais ; par la suite quand on décide de porter de 1,6 à $3,2 \mathrm{~m}$ le mouillage dans Paris, on relève le barrage de $\mathrm{Su}$ resnes de $1 \mathrm{~m}$... et on creuse l'amont du lit de la Seine de $60 \mathrm{~cm}$ sur pratiquement toute sa largeur.

(9) Le pont Notre Dame avait toutefois une heureuse particularité : c'était le pont le plus haut de Paris et ses voûtes ne devaient guère rétrécir le passage des eaux lors des grandes crues. 
- en 1885, une pile du Pont Neuf (côté petit bras) s'affaisse et l'on examine sa fondation : la plate-forme de bois supportant les piles avait été posée directement sur le fond du lit (constitué de bon "tuf") lors de la construction. "Le dessus des plateformes était arasé à des cotes variant de 24,15 à 24,36 (10)". "Ceci était très valable au moment de la construction où le fond du lit était à $25,70 . . . "$ mais il n'en est "plus de même aujourd'hui depuis que le fond normal du fleuve a été ramené à la cote 23,80 ". "[Nous] ajouterons que dans l'arche $\mathrm{n}^{\circ} 3$ le chenal était placé aux abords même de la pile $\mathrm{n}^{\circ}{ }^{2}$ " et à des cotes "variant de 22,80 à 23,00 m." Dans l'arche 2 le chenal était moins bas et plus éloigné de la pile mais il "atteignait encore la cote 23,35 ", soit 2 à $3 \mathrm{~m}$ d'approfondissement ! (11).

- le pont au Change a été reconstruit sous le Second Empire ; les maçonneries des 6 piles du pont précédent reposaient sur des planchers dont les cotes étaient comprises entre 0,05 et 1,50 (moyenne 0,61 ) sous l'étiage. Le fond de la rivière était donc à $2 \mathrm{~m}$ environ au dessus de la cote aujourd'hui garantie à la navigation.

- au pont de la Tournelle, la cote de l'étiage naturel était à 25,62 m (12), le point le plus bas du lit devait être à peu près $1,5 \mathrm{~m}$ en dessous ; aujourd'hui le plan d'eau à l'étiage est à 26,30 c'est-à-dire que la cote du fond est inférieure à 23,10 sur toute la largeur du chenal navigable.

- au banc de l'Aiguillette (vers le pont de l'Alma) le fond se situait jadis vers 23,5 ou $24 \mathrm{~m}^{(12)}$; aujourd'hui il ne dépasse pas $23,10 \mathrm{~m}$.

Seul un modèle mathématique (un modèle physique serait inutilement coûteux et guère plus efficace) pourrait permettre de préciser les conséquences de cet approfondissement mais celui-ci pourrait être du même ordre de grandeur (et de sens contraire) que les "étranglements" des XVIIlème et XVIIIİ̀me siècles.

\section{De 1910 à 1955}

A la suite de la crue de 1910 différents réaménagements ont été effectués dont on a dit, à ma connaissance sans justification et en exagérant sensiblement, qu'ils avaient permis de baisser de $60 \mathrm{~cm}$ le plan d'eau en période de crue. Trente centimètres me paraissent constituer une évaluation beaucoup plus raisonnable si l'on compare les résultats des jaugeages de crue complets et précis effectués à partir de 1955 (les premiers comportant l'exploration de la totalité de la section) avec les jaugeages du siècle antérieur qui ne mesuraient que les vitesses en surface. Mais ici aussi un modèle mathématique pourrait aujourd'hui permettre d'estimer avec plus de vraisemblance leur efficacité.

On peut rappeler à ce sujet que les mesures les plus radicales envisagées vers 1950 pour élargir le bras de la Monnaie à Paris (mesures étudiées sur modèle réduit par le L.N.H. de Chatou en 1955 et jamais mis en œuvre) n'auraient abaissé le plan d'eau à l'amont de l'lle St Louis que de $14 \mathrm{~cm}$.

\section{IV $\square$ CONCLUSION}

En commençant la rédaction de cette communication je pensais n'avoir qu'à compléter ou améliorer les nombreuses études antérieures énumérant les cotes des grandes crues des siècles précédents en regrettant une fois de plus qu'on ne puisse y associer des débits.

J'ai eu la surprise de constater que des articles ou documents anciens, n'ayant parfois aucun lien avec les crues de la Seine, permettaient par recoupement d'obtenir suffisamment d'informations pour permettre la modélisation mathématique des écoulements (ou plus exactement de leurs modifications) depuis 4 ou 5 siècles en tenant compte de l'évolution du cours de la Seine à la traversée de Paris pendant cette période.

Je n'ai pas mentionné de références dans le texte car beaucoup de mes affirmations proviennent du recoupement de plusieurs sources différentes. Je me propose d'établir un document plus détaillé où ces sources et ces recoupements seront précisées.

(10) Nivellement Bourdaloue.

(11) Je ne sais pas comment la valeur 25,70 a été déterminée : elle me parât élevée et il en est possible que l'approfondissement réel soit un peu plus faible. (12) Nivellement Lalleman.
Pour le moment, je pense que les crues de 1658 et 1910 sont équivalentes, celle de 1910 ayant peut-être eu un débit un peu supérieur. Il s'agit certainement des 2 plus grandes crues des 6 derniers siècles.

\section{BIBLIOGRAPHIE}

Les documents sont mentionnés dans l'ordre chronologique.

[1] Journal. D'un Bourgeots De Paris (au début du $15 \mathrm{e} \mathrm{sc}$ ).

[2] Journal. d'un Bourgeots de Paris (au début du 16e sc).

[3] Plumitr du Pont Neuf (1579-1606).

[4] Propositions et avis donnés en l'Hotel. de Ville de Paris pour la DÉCHARGE DES GRANDES EAUX (5.7.1651).

[5] Discours fait en l'assemblée de L'Hơtel. de Ville (24.5.1658) par Petit, intendant des fortifications.

[6] HistoIRE ET RECHERCHE DES ANTTQUTTÉS DE LA VILLE DE PARIS (antérieur à 1676) de Sauval.

[7] EXPOSÉ À L'ACADÉmIE dES SCIENCES (31.1.1720) de De Lisle le Cadet.

[8] Histoire de L'ACAdÉMIE dES SCIENCES (année 1720 page 10).

[9] DESCRIPTION D'UNE MACHINE POUR MESURER LA VITESSE DES EAUX COURANTES par Pitot (mémoires de l'Ac. des Sc. 1732).

[10] OBSERVATIONS MÉTÉoROLOGıQUES (en 1740) par Maraldi (mémoire de l'Ac. des Sc 1740).

[11] OBSERVATIONS SUR L'ÉTENDUE ET LA HAUTEUR DE L'INONDATTON DU MOIS DE DéCEMBRE 1740 par Ph. Buache (Mémoires de l'Ac. des Sc 1741).

[12] Mémotre SUR L'inoNdation de LA SeINE $\lambda$ PARIS... en 1740... par Bonamy (nov 1741) (Académie des Inscriptions et belles lettres tome XVII).

[13] MÉmOIRE SUR LES INCURSIONS QUE LES NoRMANDS FIRENT EN NEUSTRIE PAR LA SEINE par Bonamy (même référence),

[14] Exposé d'un plan hydrographique de la Ville de Paris par Ph. Buache (mai 1742) (mémoires de l'Ac. des Sc 1742).

[15] MÉmoIre SUR LA POSSIBIIITÉ d'AMENER A PARIS... LES EAUX d'ARCUEIL par Deparcieux (mémoire de l'Ac. des Sc 1762).

[16] Mémoire SUR Les INONDATIONS de LA SeINe A PARIS par Deparcieux (mémoires de l'Ac. des Sc 1764).

[17] EXPOSÉ DE DIVERS OBJETS DE GÉOGRAPHIE PHYSIQUE... par Buache (mémoire de l'Ac. des Sc 1767).

[18] Profil. RePrésentant LA CRUE... [à l'échelle de la Tournelle] par Buache (1770).

[19] Mémotre SUR L'état MOYen des EAUX de LA SEINE A Paris par De La Lande (mémoire de l'Ac. des Sc. 1788).

[20] DES FATTS ET OBSERVATIONS [sur la crue de 1802] par Bralle.

[21] MÉMOIRE SUR LES INONDATIONS DE PARIS par Egault (1814)

[22] RECHERCHES STATISTIQUES SUR LA VILE DE PARIS ET LE DépARTEMENT DE LA SEINE (1823).

[23] Statistique des variations du niveau de La Seine a Paris [de 1777 à 1825] par Dausse (1831).

[24] SUR QUELQUES ESSAIS DE DRAGAGE... par Rupelle (annales des P \& C 1842).

[25] StATISTIQUes SUR Les ÉGouts de PARIS (annales des P \& C 1836).

[26] HistoIRE DE L'Hơtel DE VILLE dE PARIS par Le Roux de Lincy (1846).

[27] ORDONNANCE CONCERNANT LE REMONTAGE DES BATEAUX [à Paris] (1848).

[28] NOTICE SUR LA DÉMOLTTION [du pont au change] par Vaudrey (annales des P \& C 1862).

[29] NOTICE HISTORIQUE SUR LES PONTS DE PARIS PAR FEIINE-ROMANY (annales des P \& C 1864)

[30] ESSAI SUR LE RÉGIME DES EAUX... en septembre 1866 par Belgrand (annales des $\mathrm{P} \& \mathrm{C}$ 1868)

[31] NOTICE SUR LE BARRAGE [de Joinville] par Malezieux (annales des P \& C 1868).

[32] NOTICE SUR LES BATEAUX-OMNIBUS DE PARIS par Lagrene (annales des P \& C 1869).

[33] NOTE SUR LA CRUE DE 1876 (annales des P \& C 1877)

[34] Notice sur les travaux de Restauration du Pont Neuf par Guiard (annales des P \& C 1891).

[35] Observations fartes SUR LA Seine A ParIs [en 1910] par Lefort et Delachenal (annales des P \& C 191i).

[36] ObServations fatres sur la Seine a Paris [en 1910] par Arana (annales des $P$ \& $C$ 1911).

[37] TRANSFORMATION EN PORTS DROrrs [des ports de tirage à Paris] par Arana (annales des P \& C 1912).

[38] Nơte SUR Les débits dE LA SeINe À PARIS par Lang (annales des P \& C 1926).

[39] Chronique d'élargissement dU PONT de LA CONCoRde (annales des P \& C 1930).

[40] LA CRUE DE LA SEINE DE JANVIER 1955 par Babinet (Mémoires et travaux de la SHF - 1955).

[41] Projet d'élargissement du bras de la Monnaie - compte rendu d'essai du LNH (1955). 